\title{
Long noncoding RNA AFAP1-AS1 enhances cell proliferation and invasion in osteosarcoma through regulating miR-4695-5p/TCF4- $\beta$-catenin signaling
}

\author{
RONGRUI LI ${ }^{1 *}$, SHICHEN LIU $^{2 *}$, YAO LI $^{1}$, QINGXI TANG ${ }^{3}$, YUNCHUAN XIE $^{1}$ and RAOSHENG ZHAI \\ ${ }^{1}$ Second Department of Orthopaedic Surgery; ${ }^{2}$ First Department of Orthopaedic Surgery; \\ ${ }^{3}$ Department of Emergency Surgery, The First Affiliated Hospital of Jiamusi University, \\ Jiamusi, Heilongjiang 154002, P.R. China
}

Received February 10, 2018; Accepted May 18, 2018

DOI: $10.3892 / \mathrm{mmr} .2018 .9131$

\begin{abstract}
Long noncoding RNA AFAP1-AS1 has been shown to promote tumor progression in several human cancer types, such as thyroid cancer, tongue squamous cell carcinoma and lung cancer. However, the role of AFAP1-AS1 in osteosarcoma (OS) has not been investigated. In the present study, the expression of AFAP1-AS1 was significantly upregulated in OS tissues and cell lines. Moreover, AFAP1-AS1 expression was negatively correlated with OS patient prognosis. Besides, AFAP1-AS1 knockdown significantly inhibited the proliferation and invasion of OS cells in vitro. Furthermore, in vivo xenograft experiments indicated that AFAP1-AS1 depletion delayed tumor growth. Regarding the underlying mechanism, AFAP1-AS1 served as a sponge to repress the level of microRNA (miR)-4695-5p, which targeted transcription factor (TCF) 4 , a pivot effector of Wnt/ $\beta$-catenin signaling pathway. It was demonstrated that overexpression of AFAP1-AS1 inhibited the expression of miR-4695-5p, while miR-4695-5p overexpression decreased TCF4 expression and reduced activation of $\mathrm{Wnt} / \beta$-catenin pathway. Through rescue assays, it was demonstrated that restoration of TCF4 expression reversed the effects of AFAP1-AS1 knockdown or miR-4695-5p overexpression on OS cells. Taken together, these findings demonstrated that the AFAP1-AS1/miR-4695-5p/TCF4- $\beta$-catenin axis played an important role in OS progression.
\end{abstract}

Correspondence to: Professor Rongrui Li, Second Department of Orthopaedic Surgery, The First Affiliated Hospital of Jiamusi University, 348 Dexiang Street, Xiangyang, Jiamusi, Heilongjiang 154002, P.R. China

E-mail: 1cr20150208@163.com

*Contributed equally

Key words: AFAP1-AS1, miR-4695-5p, TCF4, Wnt/ $\beta$-catenin, osteosarcoma

\section{Introduction}

Osteosarcoma (OS) is the most common and aggressive bona tumor that usually occurs among adolescents and young people (1). OS gives rise to many cancer-related deaths worldwide every year (2). Surgery combined with chemotherapeutic treatment is the major strategy for OS intervention (3). Despite extensive advances achieved on OS therapy in recent years, the five-year survival rate of OS patients is quite poor (4). Thus, there is an urgent need to understand the underlying mechanism involved in OS development and progression, which is crucial for the development of effectively therapeutic targets.

Long noncoding RNAs (lncRNAs) is a subgroup of noncoding RNAs that possess a length of more than 200 nucleotides $(5,6)$. In the past decade, lncRNAs have become a very hot topic in the field of biology. More and more evidences indicate that lncRNAs are involved in almost all physiological processes, such as immune, development and tumorigenesis (6-8). Due to the important functions of lncRNAs on cell proliferation, migration and invasion (9), IncRNA aberrant expression is closely correlated with the development of human cancers, including cholangiocarcinoma (10), pancreatic cancer (11), breast cancer (12), gastric cancer (13), hepatocellular carcinoma (14) and OS (8). Furthermore, several reports indicate that lncRNAs may serve as biomarkers for cancer diagnosis or prognosis (15). Therefore, understanding the functions and mechanism of lncRNAs is critical for cancer therapy.

LncRNA AFAP1-AS1 has been demonstrated to promote the development or progression of some cancers, such as thyroid cancer (16) and tongue squamous cell carcinoma (17). Whether AFAP1-AS1 has a role in OS progression remains unclear. In the present study, we found that AFAP1-AS1 expression was significantly upregulated in OS tissues and cell lines. AFAP1-AS1 high expression is linked to OS patients' poor prognosis. Moreover, we showed that AFAP1-AS1 knockdown significantly suppressed the proliferation and invasion of OS cells. Mechanistically, we found that AFAP1-AS1 sponged microRNA (miR)-4695-5p to upregulate the expression of transcription factor (TCF)4, which is a pivot transcription factor in $\mathrm{Wnt} / \beta$-catenin pathway. Taken together, our results demonstrated that 
AFAP1-AS1/miR-4695-5p/TCF4/ $\beta$-catenin signaling cascade was involved in OS progression.

\section{Materials and methods}

Clinical specimens. A total of 49 samples of OS tissues and adjacent normal tissues were obtained from patients who underwent surgery at The First Affiliated Hospital of Jiamusi University (Jiamusi, China). Two pathologists independently evaluated the histological diagnosis and differentiation of the tissue samples, according to the World Health Organization classification system. All of the tissue samples collected were immediately snap-frozen in liquid nitrogen and stored at $-80^{\circ} \mathrm{C}$ until required. The present study was approved by the Research Ethics Committee of The First Affiliated Hospital of Jiamusi University, and informed consent was obtained from all patients.

Cell culture and transfection. Normal human osteoblasts, hFOB 1.19, and four human OS cell lines, Saos-2, U2OS, MG-63 and 143B, were purchased from American Type Culture Collection (Manassas, VA, USA). Cell lines were cultured in DMEM (Gibco; Thermo Fisher Scientific, Inc., Waltham, MA, USA) supplemented with $10 \%$ fetal bovine serum (FBS; Gibco; Thermo Fisher Scientific, Inc.) and 100 units/ml of penicillin-streptomycin (Invitrogen; Thermo Fisher Scientific, Inc.) at $37^{\circ} \mathrm{C}$ in a humidified incubator containing $5 \% \mathrm{CO}_{2}$.

A siRNA against AFAP1-AS1 (named as si-AFAP1-AS1), corresponding scramble negative control (NC) (named as scramble), miR-4695-5p mimics, mimic NCs were designed and constructed by Ribobio (Guangzhou, China). The target sequence was as followed: si-AFAP1-AS1: 5'-AGGACACAG ACUGCUUCAU-3'; and scramble: 5'-UUCUCCGAACGU GACGUTT-3'. AFAP1-AS1 and TCF4 overexpression plasmids (pCDNA3.1-AFAP1-AS1 or pCDNA3.1-TCF4) were cloned into pCDNA3.1 Vector. The sequences were determined by DNA sequencing. These plasmids were transfected into OS cells in 6-well plates at a final concentration of $100 \mathrm{ng}$ using Lipofectamine ${ }^{\circledR} 2000$ (Invitrogen; Thermo Fisher Scientific, Inc.) according to the manufacturer's protocol.

Cell proliferation assay. For cell proliferation assays, the viable cells were tested by Cell Counting Kit-8 (CCK-8) assay kit according to the manufacturer's instructions. In brief, cells were grown in 96-well plate with $1 \times 10^{4}$ per well and incubated in $37^{\circ} \mathrm{C}$ with $5 \% \mathrm{CO}_{2}$ until cell confluent rate reached $70 \%$. After transfected with plasmid for $48 \mathrm{~h}$, cells were still incubated for 24,48 and 72 h. $10 \mu \mathrm{l} \mathrm{CCK}-8$ solution was seed into each well. The absorbance at $450 \mathrm{~nm}$ was measured with SUNRISE Microplate Reader (Tecan Group, Ltd., Mannedorf, Switzerland).

Transwell invasion assay. Cell invasion was assayed using a Transwell invasion assay (invasion Transwell chambers; EMD Millipore, Billerica, MA, USA) with Matrigel $^{\mathrm{TM}}$ (BD Biosciences). The cells were seeded at a density of $1 \times 10^{5}$ cells on the upper chamber with $200 \mu 1$ serum-free DMEM. Following transfection for $48 \mathrm{~h}, 600 \mu \mathrm{l}$ DMEM supplemented with $20 \%$ FBS, which served as a chemoattractant, was added to the lower chamber. After $48 \mathrm{~h}$ incubation at $37^{\circ} \mathrm{C}$, the upper side of the membrane was wiped with cotton wool to remove non-invasive cells; the membranes were then fixed with $4 \%$ methanol at $4^{\circ} \mathrm{C}$ and stained with $0.1 \%$ crystal violet at room temperature. Five visual fields with a magnification x 200 were randomly selected from each membrane and the cell numbers were counted using a light microscope.

Reverse transcription-quantitative polymerase chain reaction $(R T-q P C R)$. RNA was isolated using TRIzol reagent (Invitrogen; Thermo Fisher Scientific, Inc.) following the manufactures' protocol. The first strand cDNA was compounded using a Tianscript RT kit (Tiangen Biotech, Beijing, China). PCR amplification was performed using TaqMan Human MicroRNA Assay (Applied Biosystems; Thermo Fisher Scientific, Inc.) and UltraSYBR Mixture (CW0957; CWbio, Beijing, China) in LC 480 PCR System (Roche Diagnostics, Indianapolis, IN, USA). U6 and $18 \mathrm{~S}$ was employed as reference genes to normalize the expression of miR-4695-5p or AFAP1-AS1 and TCF4. The primers used were synthesized and purchased from Genecopoeia (Guangzhou, China). Relative expression level was analyzed on the basis of the $2^{-\Delta \Delta \mathrm{Cq}}$ method (18).

Tumor xenograft. 4-6-weeks old female BALB/c nude mice were used for all in vivo xenograft studies. All animal experiments were approved by the Animal Care and Use Committee of The First Affiliated Hospital of Jiamusi University. Cells were injected subcutaneously into the flanks of nude mice $\left(2 \times 10^{6}\right.$ cells per animal). All mice were sacrificed 35 days after seeding of tumor cells, and the tumor weights measured.

Luciferase assays. Wild-type (WT) or mutant (Mut) fragment (801-1,660 bp) of AFAP1-AS1 containing the predicted miR-4695-5p binding sites were designed, constructed and cloned by Sangon Biotech (Shanghai, China) downstream of the firefly luciferase gene in the pGL3-control vector (Promega Corporation, Madison, WI, USA) to form pGL3-AFAP1-AS1-WT and pGL3-AFAP1-AS1-Mut, respectively.

For the luciferase reporter assays, OS cells were seeded into 12-well plate cells and co-ransfected with $300 \mathrm{ng}$ of pGL3-AFAP1-AS1-WT or pGL3-AFAP1-AS1-Mut and $50 \mathrm{nM}$ of miR-4695-5p or NC using Lipofectamine 2000 (Invitrogen; Thermo Fisher Scientific, Inc.). Cells were collected $48 \mathrm{~h}$ after transfection, and luciferase activity was measured using a dual-luciferase reporter assay system (Promega Corporation). The firefly luciferase activity of each sample was normalized to the Renilla luciferase activity of each sample.

Statistical analysis. Each experiment was repeated at least three times. All data are expressed in terms of mean \pm standard deviation. The Kaplan-Meier method was used to calculate the survival curve, and log-rank test to determine statistical significance. Student's t-test and one-way ANOVA followed by Tukey's post hoc test were used to analyze 2 or multiple groups, respectively, for statistical significance. Pearson correlation coefficient analysis was used to determine the correlations. $\mathrm{P}<0.05$ was considered to indicate a statistically significant difference. 


\section{Results}

AFAPI-AS1 is highly expressed in OS tissues. Firstly, we analyzed the expression patterns of AFAP1-AS1 in OS tissues by RT-qPCR. We examined the levels of AFAP1-AS1 in 49 OS tissues and paired adjacent normal tissues, and found that the expression of AFAP1-AS1 was significantly upregulated in OS tissues compared to adjacent normal tissues (Fig. 1A). Consistently, the expression of AFAP1-AS1 was also upregulated in OS cell lines, including Saos-2, U2OS, MG-63 and 143B cells, compared with hFOB1.19 cells (Fig. 1B). To analyze whether AFAP1-AS1 could serve as a prognostic marker for OS patients, we performed Kaplan-Meier survival curve analysis, and found that higher expression of AFAP1-AS1 in OS patients showed lower overall and progression-free survival rates (Fig. 1C and D).

AFAPI-AS1 depletion suppresses the proliferation and invasion of OS cells. To explore the function of AFAP1-AS1 in OS cells, we silenced AFAP1-AS1 in Saos-2 and U2OS cells. RT-qPCR analysis indicated that AFAP1-AS1 expression was significantly downregulated in Saos-2 and U2OS cells transfected with siAFAP1-AS1 (Fig. 2A). Then CCK-8 assays were used to analyze the effect of AFAP1-AS1 knockdown on cell proliferation. As shown, knockdown of AFAP1-AS1 significantly inhibited the proliferation of Saos-2 and U2OS cells (Fig. 2B and C). Due to the correlation between cancer cell metastasis and tumor malignance, we then determined the effect of AFAP1-AS1 on tumor cell metastasis. Through transwell invasion assays, we found that AFAP1-AS1 knockdown significantly reduced the numbers of invaded Saos-2 and U2OS cells (Fig. 2D). To further determine the effect of AFAP1-AS1 on tumor growth in vivo, we conducted a xenograft experiment. We injected AFAP1-AS1-silenced or control Saos-2 cells into nude recipient mice. After 5 weeks, we sacrificed these mice and measured tumor weights. The results indicated that knockdown of AFAP1-AS1 significantly decreased the tumor size (Fig. 2E). Taken together, our results demonstrated that AFAP1-AS1 serves as an oncogene to regulate OS cell proliferation and invasion.

AFAPI-AS1 suppresses the expression of miR-4695-5p in OS cells. LncRNAs have been shown to sponge miRNAs and regulate gene expression. In order to determine the mechanism of AFAP1-AS1 in OS cells, we explored the target miRNAs. Through bioinformatics analysis, we found that miR-4695-5p was the most potential candidate. We found that there were three potential binding sites of miR-4695-5p in AFAP1-AS1 (Fig. 3A). To verify their interaction, we conducted luciferase reporter assays using WT or Mut AFAP1-AS1 reporter plasmid. We first confirmed the overexpression of miR-4695-5p in Saos-2 and U2OS cells after transfection (Fig. 3B). Furthermore, overexpression of miR-4695-5p significantly inhibited the luciferase activity in Saos-2 cells transfected with WT-AFAP1-AS1, whereas mutation of these binding sites abrogated this effect (Fig. 3B). Furthermore, RT-qPCR analysis indicated that overexpression of miR-4695-5p markedly suppressed the expression of AFAP1-AS1 in Saos-2 and U2OS cells (Fig. 3C). In addition, we found that knockdown of AFAP1-AS1 also promoted the levels of miR-4695-5p in Saos-2
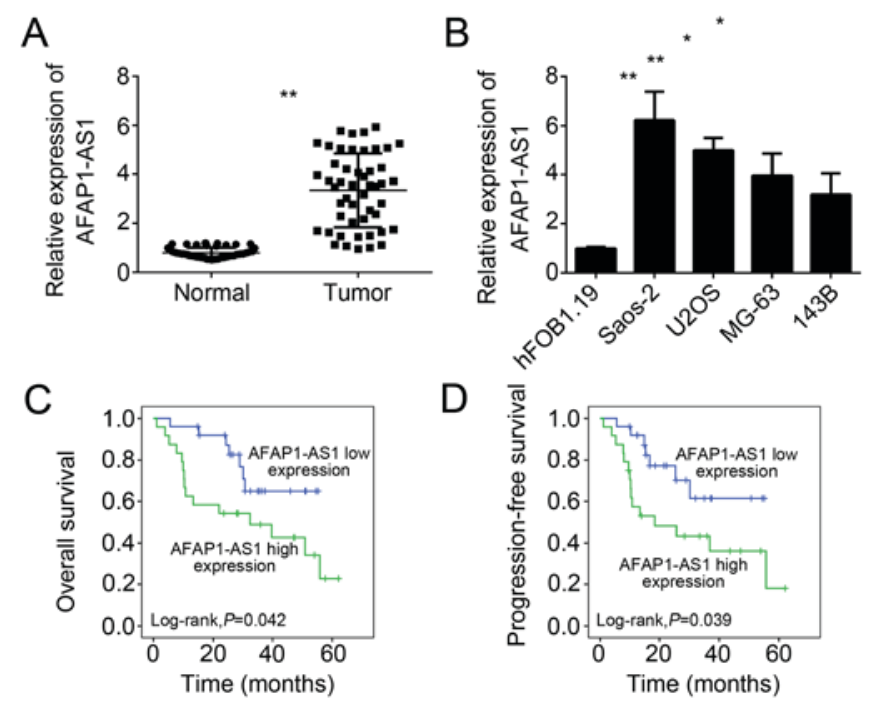

Figure 1. AFAP1-AS1 was highly expressed in OS tissues. (A) Relative expression levels of AFAP1-AS1 in 49 paired OS tissues and adjacent normal tissues. (B) RT-qPCR analysis showed that AFAP1-AS1 was upregulated in OS cell lines compared to hFOB1.19 cells. (C and D) Kaplan-Meier analysis was conducted to measure the probabilities of overall and progression-free survival in OS patients based on AFAP1-AS1 expression levels. The results represented three independent experiments and were expressed as mean $\pm \mathrm{SD}$. ${ }^{*} \mathrm{P}<0.05$ and ${ }^{* *} \mathrm{P}<0.01$ vs. the control group. OS, osteosarcoma; RT-qPCR, reverse transcription-quantitative polymerase chain reaction.

and U2OS cells (Fig. 3D). Then we overexpressed AFAP1-AS1 and overexpression of AFAP1-AS1 reduced the expression of miR-4695-5p in Saos-2 and U2OS cells (Fig. 3E). Summarily, above data indicated that AFAP1-AS1 sponged miR-4695-5p to regulate its expression in OS cells.

TCF4 is a direct target of miR-4695-5p in OS cells. To further explore the downstream mechanism of miR-4695-5p, we searched the target genes of miR-4695-5p. Using TargetScan website, we found that TCF4 was one of potential target genes of miR-4695-5p in OS cells. TCF4 is a pivot transcription factor of Wnt/ $\beta$-catenin pathway and involved in tumor progression in various cancers. Therefore, we chose TCF4 for further investigation. We also found that there was a potential binding site of miR-4695-5p in the 3'-URT region of TCF4 mRNA (Fig. 4A). Luciferase reporter assays indicated that overexpression of miR-4695-5p significantly repressed the luciferase activity in Saos-2 cells transfected with WT-TCF4-3'-UTR (Fig. 4B). Besides, RT-qPCR showed that overexpression of miR-4695-5p inhibited the mRNA levels of TCF4 in Saos-2 and U2OS cells (Fig. 4C). Moreover, there was a reversely correlation between the expression of miR-4695-5p and TCF4 in OS tissues (Fig. 4D). Then we performed western blot to analyze the activation of $\mathrm{Wnt} / \beta$-catenin pathway. Cyclin D1 and MYC are two classical target genes of Wnt/ $\beta$-catenin pathway. Therefore, we chose them as markers for evaluation of $\mathrm{Wnt} / \beta$-catenin pathway activation. The results indicated that both overexpression of miR-4695-5p and AFAP1-AS1 knockdown inhibited the protein levels of TCF4, Cyclin D1 and MYC in Saos-2 and U2OS cells (Fig. 4E). Taken together, our results indicated that miR-4695-5p and AFAP1-AS1 regulated TCF4 expression and Wnt/ $\beta$-catenin pathway activation in OS cells. 

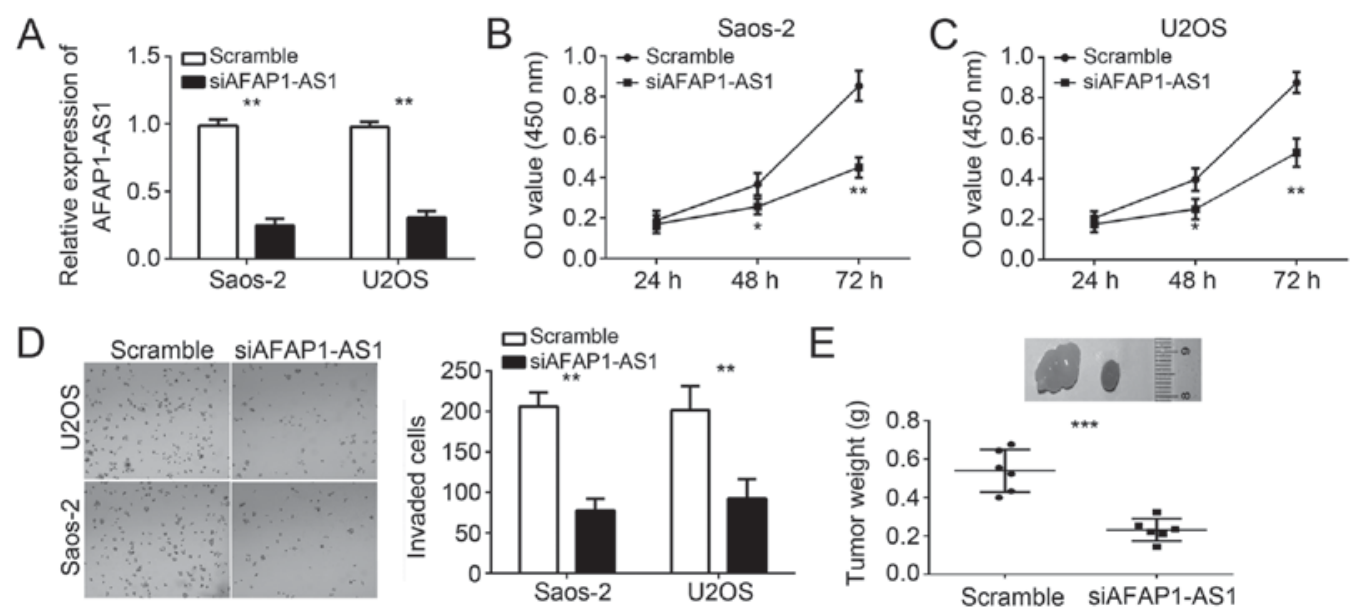

Figure 2. AFAP1-AS1 depletion suppressed the proliferation and invasion of OS cells. (A) RT-qPCR was utilized to check the expression of AFAP1-AS1 in Saos-2 and U2OS cells transfected with scramble or siAFAP1-AS1. (B and C) CCK-8 assay indicated that AFAP1-AS1 knockdown inhibited the proliferation of Saos-2 and U2OS cells. (D) Transwell assays indicated that AFAP1-AS1 knockdown reduced the invasion of Saos-2 and U2OS cells. (x100, magnifications). (E) Tumor weights were determined at the endpoint of xenograft experiments. The results represented three independent experiments and were expressed as mean $\pm \mathrm{SD} .{ }^{*} \mathrm{P}<0.05,{ }^{* *} \mathrm{P}<0.01$ and ${ }^{* * * *} \mathrm{P}<0.001$ vs. the control group. OS, osteosarcoma; RT-qPCR, reverse transcription-quantitative polymerase chain reaction; CCK-8, Cell Counting Kit-8.

A

AFAP1-AS1: 5'-----GCCTCCT---GCCTCC--GCCTCCT---3'

miR-4695-5p: 3'-GGACGAGCGGGUGACGGAGGAC-5'

AFAP1-AS1: 5'------CGGAGGA---CGGAGGA--CGGAGGA---3'

MUT MUT MUT
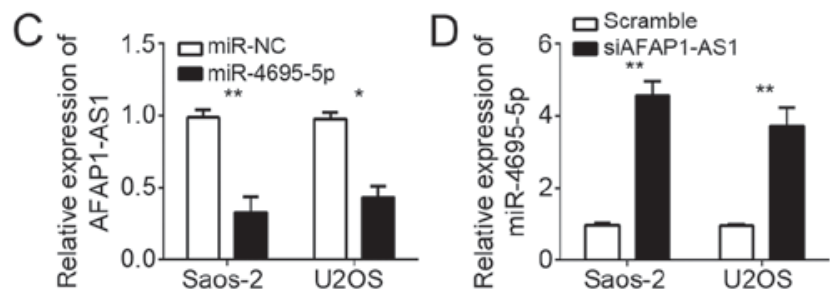
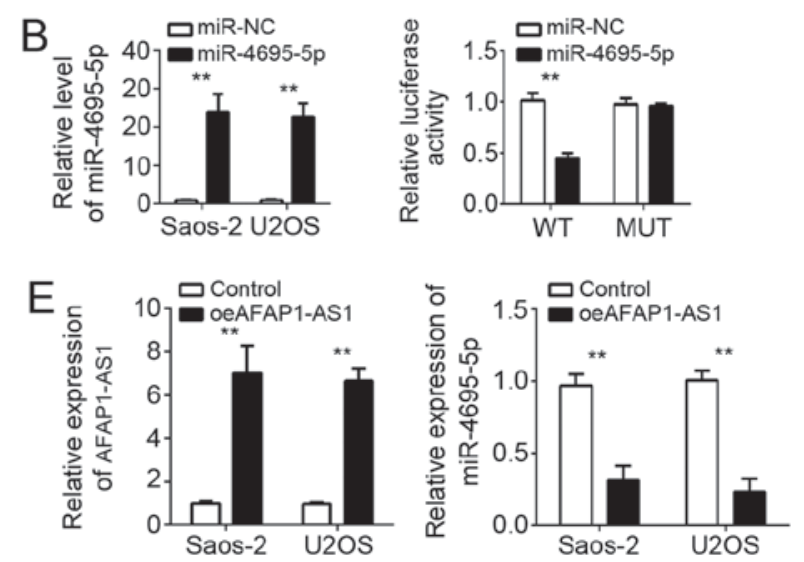

Figure 3. AFAP1-AS1 suppressed the expression of miR-4695-5p in OS cells. (A) Four potential binding sites of miR-4695-5p were shown in AFAP1-AS1. (B) RT-qPCR analysis for miR-4695-5p expression in Saos-2 and U2OS cells, followed by luciferase reporter assays using WT and Mut AFAP1-AS1 reporter in Saos-2 cells. (C) RT-qPCR analysis indicated that overexpression of miR-4695-5p suppressed the expression of AFAP1-AS1 in Saos-2 and U2OS cells. (D) RT-qPCR results showed that knockdown of AFAP1-AS1 increased the levels of AFAP1-AS1 and miR-4695-5p in Saos-2 and U2OS cells. (E) RT-qPCR analysis was used to determine the levels of miR-4695-5p in Saos-2 and U2OS cells transfected with AFAP1-AS1 or empty vector. The results represented three independent experiments and were expressed as mean $\pm \mathrm{SD}$. ${ }^{*} \mathrm{P}<0.05$ and ${ }^{* *} \mathrm{P}<0.01$ vs. the control group. OS, osteosarcoma; RT-qPCR, reverse transcription-quantitative polymerase chain reaction; WT, wild-type; Mut, mutant.

AFAP1-AS1 promotes $O S$ cell proliferation and invasion by regulating activation of TCF4/ $\beta$-catenin pathway. Then we analyzed the expression of TCF4 in OS tissues and found that TCF4 expression was significantly upregulated in OS tissues compared with adjacent normal tissues (Fig. 5A). To further confirm that TCF4 is indispensable for the role of AFAP1-AS1 in OS cells, we restored the protein level of TCF4 in Saos2 and U2OS cells transfected with siAFAP1-AS1 or miR-4695-5p mimics. RT-qPCR and Western blot showed that the expression of TCF4 was significantly upregulated in these two cells (Fig. 5B). Moreover, CCK-8 and transwell invasion assays indicated that miR-4695-5p overexpression or AFAP1-AS1 knockdown inhibited cell proliferation and invasion, while restoration of TCF4 significantly rescued the proliferation and invasion of Saos-2 and U2OS cells (Fig. 5C and D). In conclusion, these data indicated that AFAP1-AS1 promoted OS cell proliferation and invasion by regulating miR-4695-5p/TCF4/ $\beta$-catenin signaling.

\section{Discussion}

LncRNA AFAP1-AS1 has been demonstrated to be overexpressed and act as an oncogene in several cancers $(16,17)$. For instance, Han et al reported that AFAP1-AS1 is overexpressed in colorectal cancer, and promotes tumor growth and metastasis (19). Zhang et al reported that upregulated AFAP1-AS1 expression promotes hepatocellular carcinoma cell proliferation and invasion, and predicts a poor prognosis (20). In addition, Deng et al showed that AFAP1-AS1 overexpression is associated with a poor prognosis in NSCLC patients (21). Nevertheless, the function of AFAP1-AS1 in OS remains largely unknown. In this study, we found that 
A

TCF4:5'-GUAGAUGACUUAACUGCCUCCUC-3'

miR-4695-5p: 3'-GGACGAGCGGGUGACGGAGGAC-5'

TCF4:5'-GUAGAUGACUUAAGACGGAGGUC--3'

MUT
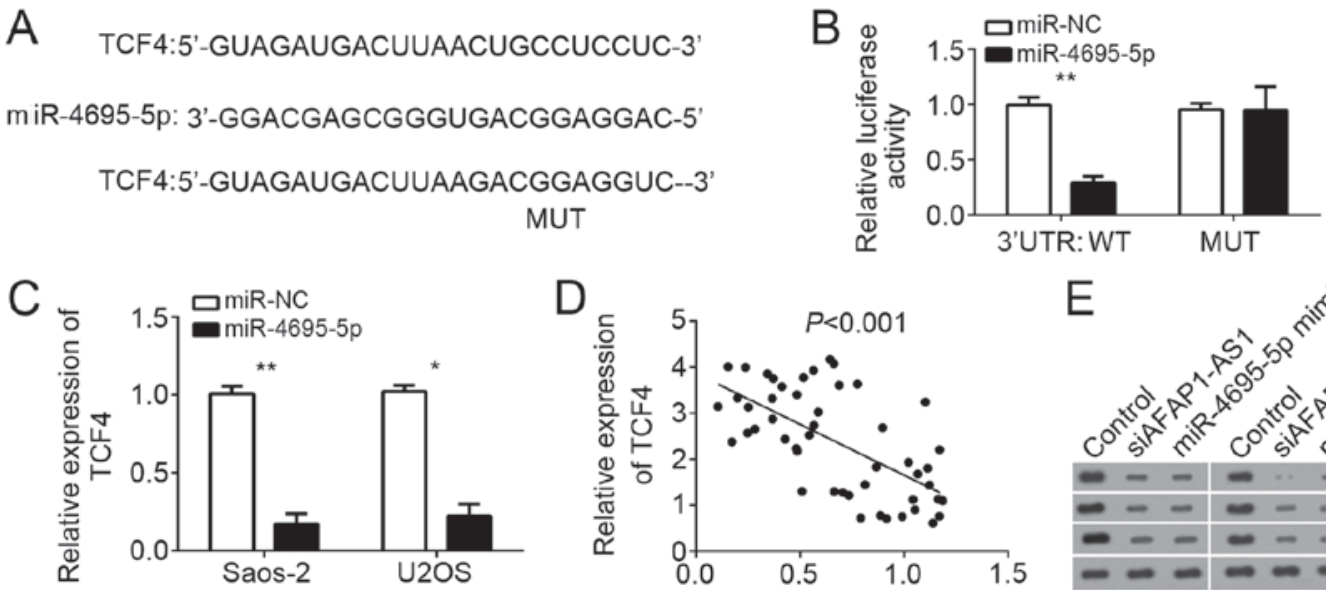

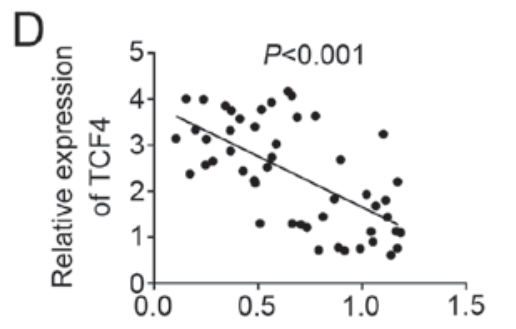

Relative expression of miR-4695-5p

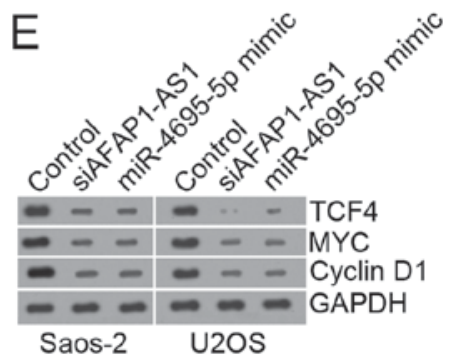

Figure 4. TCF4 was a direct target of miR-4695-5p in OS cells. (A) Predicted binding site of miR-4695-5p in the 3'-UTR of TCF4 mRNA. (B) Luciferase reporter assays indicated that overexpression of miR-4695-5p suppressed the luciferase activity in Saos-2 cells transfected with WT 3'-UTR. (C) RT-qPCR analysis indicated that overexpression of miR-4695-5p inhibited the mRNA levels of TCF4 in Saos-2 and U2OS cells. (D) RT-qPCR analysis showed that the expression of miR-4695-5p was reversely correlated with that of TCF4 in OS tissues. (E) Overexpression of miR-4695-5p suppressed the protein level of TCF4 and activation of Wnt/B-catenin pathway in Saos-2 and U2OS cells. The protein level of TCF4, Cyclin D1 and MYC were measured by western blot. GAPDH was chose as a loading control. The results represented three independent experiments and were expressed as mean \pm SD. ${ }^{*} \mathrm{P}<0.05$ and ${ }^{* * *} \mathrm{P}<0.01$ vs. the control group. TCF4, transcription factor 4; OS, osteosarcoma; RT-qPCR, reverse transcription-quantitative polymerase chain reaction.
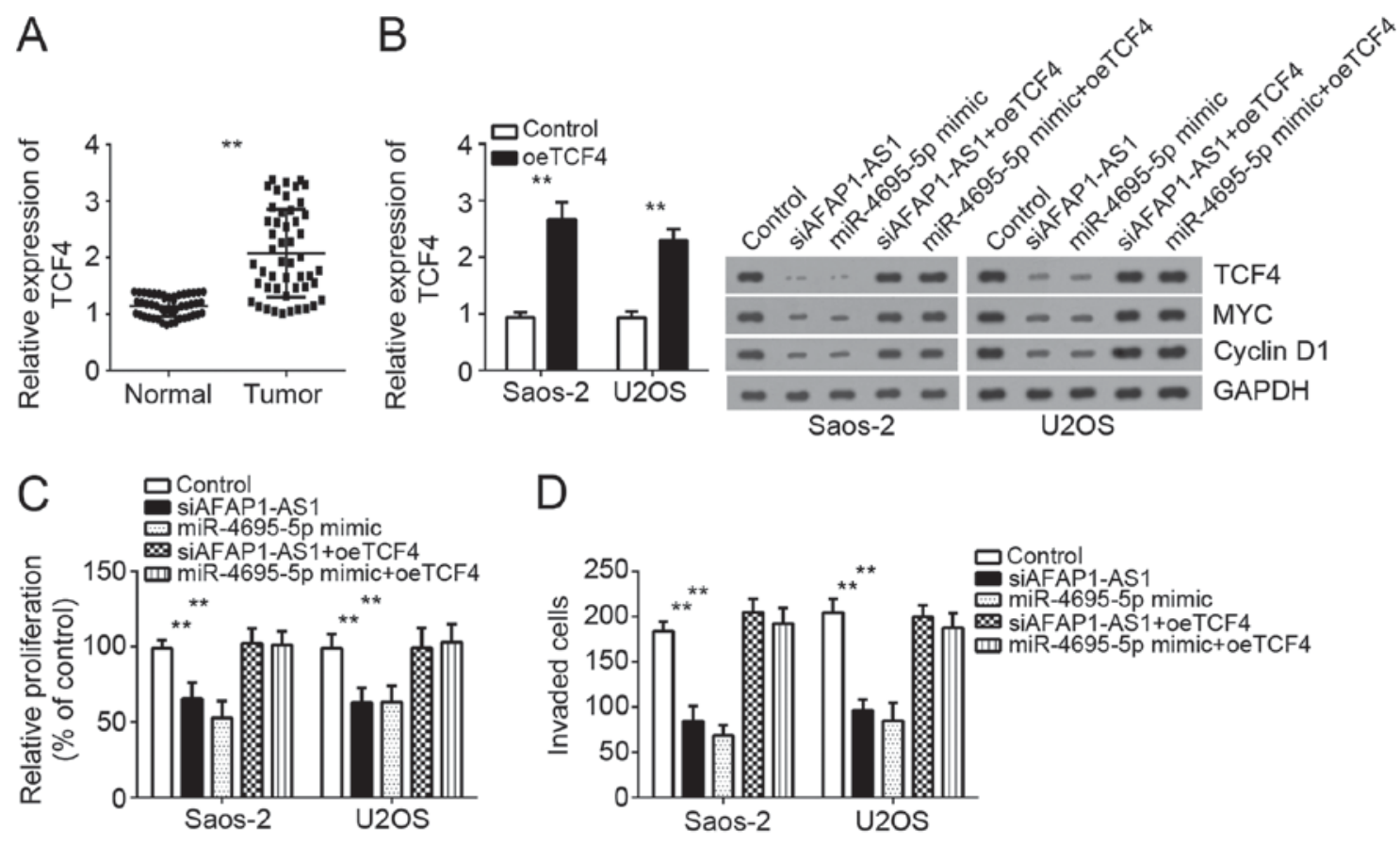

Figure 5. AFAP1-AS1 promoted OS cell proliferation and invasion by regulating activation of TCF4/ß-catenin pathway. (A) RT-qPCR was used to determine the expression of TCF4 in 58 paired OS tissues and adjacent normal tissues. (B) RT-qPCR analysis indicated TCF4 expression was significantly upregulated after transfection and western blot indicated that ectopic expression of TCF4 restored the TCF4 protein level and Wnt/ $\beta$-catenin pathway activation in Saos-2 and U2OS cells transfected with siAFAP1-AS1 or miR-4695-5p mimics. (C) CCK-8 assays were used to measure the proliferation of Saos-2 and U2OS cells. (D) Transwell assays were utilized to determine the invasion of Saos-2 and U2OS cells. The results represented three independent experiments and were expressed as mean $\pm \mathrm{SD}$. ${ }^{* *} \mathrm{P}<0.01$ vs. the control group. TCF4, transcription factor 4; OS, osteosarcoma; RT-qPCR, reverse transcription-quantitative polymerase chain reaction; CCK-8, Cell Counting Kit-8.

AFAP1-AS1 was significantly upregulated in OS tissues and cell lines. Moreover, AFAP1-AS1 overexpression predicted a poor prognosis in OS patients. Furthermore, functional experiments indicated that loss of AFAP1-AS1 expression led to reduced proliferation and invasion of OS cells in vitro. We also showed that AFAP1-AS1 knockdown resulted in delayed tumor growth in vivo. Taken together, our data indicated that AFAP1-AS1 functions as an oncogene and might be involved in OS development and progression.

Accumulating evidences indicate that lncRNAs could regulate gene expression through various mechanisms, including RNA decay, chromatin remodeling and miRNA 
sponging $(22,23)$. It was reported that AFAP1-AS1 regulates PTEN/AKT pathway to enhance gastric cell proliferation (23). Wang et al reported Upregulation of AFAP1-AS1 promotes the proliferation, invasion and survival of tongue squamous cell carcinoma cell by activation of $\mathrm{Wnt} / \beta$-catenin signaling pathway (17). However, how AFAP1-AS1 activates these signaling pathway remains unclear. And the underlying molecular mechanism of AFAP1-AS1 in cancer cells requires to be investigated. In this study, our results indicated that AFAP1-AS1 could directly associate with miR-4695-5p to serve as a competing endogenous RNA. Though luciferase reporter assays and RT-qPCR analysis, we demonstrated their direct interaction. miRs have been widely acknowledged as essential regulators in human cancers, including OS (24). miRs could bind to the complementary sequence in the 3'-UTR region of target mRNAs and regulate gene expression (25). Until now, the function of miR-4695-5p remains totally unclear. Therefore, to determine the role of miR-4695-5p in OS and whether AFAP1-AS1 promoted OS cell proliferation and invasion by sponging miR-4695-5p, we further investigated the downstream mechanism of miR-4695-5p. Through bioinformatics analysis and functional experiments, we demonstrated TCF4 was a direct target of miR-4695-5p in OS cells, which suggested that miR-4695-5p might act as a tumor suppressor via targeting TCF4.

After initiation of $\mathrm{Wnt} / \beta$-catenin signaling, $\beta$-catenin translocates to the nucleus and binds to TCF4, which leads to transcriptional activation of downstream oncogenic genes, such as MYC and Cyclin D1, by $\beta$-catenin-TCF4 complex (26). Aberrant activation of this signaling has been observed in many cancers, including colorectal cancer (27), esophageal squamous cell carcinoma (28), clear cell renal cell carcinoma (29) and lung cancer (30). The knowledge about how TCF4 expression is regulated is limited. In our study, we demonstrated AFAP1-AS1/miR-4695-5p axis regulated the expression of TCF4 and consequently influenced the activation of Wnt/ $\beta$-catenin pathway in OS cells. Moreover, we showed that knockdown of AFAP1-AS1 or miR-4695-5p overexpression suppresses OS cell proliferation and invasion. However, restoration of TCF4 protein levels significantly rescued the proliferation and invasion of OS cells transfected with siAFAP1-AS1 or miR-4695-5p mimics. These data suggested that upregulation of TCF4 by AFAP1-AS1/miR-4695-5p axis promoted OS progression.

In conclusion, our findings demonstrate that AFAP1-AS1 is overexpressed in OS tissues and associated with poor prognosis of OS patients. AFAP1-AS1 promotes the proliferation and invasion of OS cells through inhibition of miR-4695-5p and activation of TCF4- $\beta$-catenin signaling. Our data elucidated a potential mechanism underlying the tumor-oncogenic role of AFAP1-AS1 in OS.

\section{Acknowledgements}

Not applicable.

\section{Funding}

No funding was received.

\section{Availability of data and materials}

All data generated or analyzed during this study are included in this published article.

\section{Authors' contributions}

RL and SL initiated, designed this work, analyzed, interpreted the results and wrote this manuscript. YL, QT, YX and RZ performed certain experiments. All authors read and approved the final manuscript.

\section{Ethics approval and consent to participate}

For the use of human samples, the protocol for this study was approved by the Institutional Ethics Committee of The First Affiliated Hospital of Jiamusi University and all enrolled patients signed a written informed consent document. In addition, all procedures involving animals conformed to the national guidelines of and were approved by the Animal Care Ethics Committee of The First Affiliated Hospital of Jiamusi University.

\section{Consent for publication}

All patients within this study provide consent for the publication of their data.

\section{Competing interests}

The authors declare that they have no competing interests.

\section{References}

1. Messerschmitt PJ, Garcia RM, Abdul-Karim FW, Greenfield EM and Getty PJ: Osteosarcoma. J Am Acad Orthop Surg 17: 515-527, 2009.

2. Yu W, Tang L, Lin F, Yao Y, Shen Z and Zhou X: High-intensity focused ultrasound: noninvasive treatment for local unresectable recurrence of osteosarcoma. Surg Oncol 24: 9-15, 2015.

3. Bacci G, Ferrari S, Bertoni F, Ruggieri P, Picci P, Longhi A, Casadei R, Fabbri N, Forni C, Versari M and Campanacci M: Long-term outcome for patients with nonmetastatic osteosarcoma of the extremity treated at the istituto ortopedico rizzoli according to the istituto ortopedico rizzoli/osteosarcoma-2 protocol: An updated report. J Clin Oncol 18: 4016-4027, 2000.

4. Bielack SS, Kempf-Bielack B, Delling G, Exner GU, Flege S, Helmke K, Kotz R, Salzer-Kuntschik M, Werner M, Winkelmann W, et al: Prognostic factors in high-grade osteosarcoma of the extremities or trunk: An analysis of 1,702 patients treated on neoadjuvant cooperative osteosarcoma study group protocols. J Clin Oncol 20: 776-790, 2002.

5. Gutschner T and Diederichs S: The hallmarks of cancer: A long non-coding RNA point of view. RNA Biol 9: 703-719, 2012.

6. Ye B, Liu B, Yang L, Zhu X, Zhang D, Wu W, Zhu P, Wang Y, Wang S, Xia P, et al: LncKdm2b controls self-renewal of embryonic stem cells via activating expression of transcription factor Zbtb3. EMBO J 37: pii: e97174, 2018.

7. Liu B, Ye B, Yang L, Zhu X, Huang G, Zhu P, Du Y, Wu J, Qin X, Chen R, et al: Long noncoding RNA $\operatorname{lncKdm} 2 \mathrm{~b}$ is required for ILC3 maintenance by initiation of Zfp292 expression. Nat Immunol 18: 499-508, 2017.

8. Yang C, Wu K, Wang S and Wei G: Long non-coding RNA XIST promotes osteosarcoma progression by targeting YAP via miR-195-5p. J Cell Biochem: Jan 31, 2018 (Epub ahead of print).

9. Dong X, Chen R, Lin H, Lin T and Pan S: IncRNA BG981369 inhibits cell proliferation, migration, and invasion, and promotes cell apoptosis by SRY-related high-mobility group box 4 (SOX4) signaling pathway in human gastric cancer. Med Sci Monit 24: 718-726, 2018. 
10. Bai JG, Tang RF, Shang JF, Qi S, Yu GD and Sun C: Upregulation of long non-coding RNA CCAT2 indicates a poor prognosis and promotes proliferation and metastasis in intrahepatic cholangiocarcinoma. Mol Med Rep 17: 5328-5335, 2018.

11. Cheng Y, Imanirad P, Jutooru I, Hedrick E, Jin UH, Rodrigues Hoffman A, Leal de Araujo J, Morpurgo B, Golovko A and Safe S: Role of metastasis-associated lung adenocarcinoma transcript-1 (MALAT-1) in pancreatic cancer. PLoS One 13: e0192264, 2018

12. Zhang $\mathrm{P}$, Zhou H, Lu K, Lu Y, Wang $\mathrm{Y}$ and Feng T: Exosome-mediated delivery of MALAT1 induces cell proliferation in breast cancer. Onco Targets Ther 11: 291-299, 2018.

13. Chen X, Chen Z, Yu S, Nie F, Yan S, Ma P, Chen Q, Wei C, $\mathrm{Fu} \mathrm{H}, \mathrm{Xu}$ T, et al: Long noncoding RNA LINC01234 functions as a competing endogenous RNA to regulate CBFB expression by sponging miR-204-5p in gastric cancer. Clin Cancer Res 24: 2002-2014, 2018

14. Zhu P, Wang Y, Wu J, Huang G, Liu B, Ye B, Du Y, Gao G, Tian Y, He L and Fan Z: LncBRM initiates YAP1 signalling activation to drive self-renewal of liver cancer stem cells. Nat Commun 7: 13608, 2016.

15. Ren J, Yang Y, Xue J, Xi Z, Hu L, Pan SJ and Sun Q: Long noncoding RNA SNHG7 promotes the progression and growth of glioblastoma via inhibition of miR-5095. Biochem Biophys Res Commun 496: 712-718, 2018.

16. Dai W, Tian Y, Jiang B and Chen W: Down-regulation of long non-coding RNA AFAP1-AS1 inhibits tumor growth, promotes apoptosis and decreases metastasis in thyroid cancer. Biomed Pharmacother 99: 191-197, 2018.

17. Wang ZY, Hu M, Dai MH, Xiong J, Zhang S, Wu HJ, Zhang SS and Gong ZJ: Upregulation of the long non-coding RNA AFAP1-AS1 affects the proliferation, invasion and survival of tongue squamous cell carcinoma via the $\mathrm{Wnt} / \beta$-catenin signaling pathway. Mol Cancer 17: 3, 2018

18. Livak KJ and Schmittgen TD: Analysis of relative gene expression data using real-time quantitative PCR and the 2(-Delta Delta C(T)) method. Methods 25: 402-408, 2001

19. Han X, Wang L, Ning Y, Li S and Wang Z: Long non-coding RNA AFAP1-AS1 facilitates tumor growth and promotes metastasis in colorectal cancer. Biol Res 49: 36, 2016.

20. Zhang JY, Weng MZ, Song FB, Xu YG, Liu Q, Wu JY, Qin J, Jin T and Xu JM: Long noncoding RNA AFAP1-AS1 indicates a poor prognosis of hepatocellular carcinoma and promotes cell proliferation and invasion via upregulation of the RhoA/Rac2 signaling. Int J Oncol 48: 1590-1598, 2016.
21. Deng J, Liang Y, Liu C, He S and Wang S: The up-regulation of long non-coding RNA AFAP1-AS1 is associated with the poor prognosis of NSCLC patients. Biomed Pharmacother 75: 8-11, 2015.

22. Marchese FP, Raimondi I and Huarte M: The multidimensional mechanisms of long noncoding RNA function. Genome Biol 18: 206, 2017.

23. Zhu P, Wang Y, Huang G, Ye B, Liu B, Wu J, Du Y, He L and Fan Z: lnc- $\beta$-Catm elicits EZH2-dependent $\beta$-catenin stabilization and sustains liver CSC self-renewal. Nat Struct Mol Biol 23: 631-639, 2016.

24. Lin T, Ma Q, Zhang Y, Zhang H, Yan J and Gao C: MicroRNA-27a functions as an oncogene in human osteosarcoma by targeting CCNG1. Oncol Lett 15: 1067-1071, 2018.

25. Han C and Wang W: MicroRNA-129-5p suppresses cell proliferation, migration and invasion via targeting ROCK1 in osteosarcoma. Mol Med Rep 17: 4777-4784, 2018.

26. Morin PJ, Sparks AB, Korinek V, Barker N, Clevers H, Vogelstein B and Kinzler KW: Activation of beta-catenin-Tcf signaling in colon cancer by mutations in beta-catenin or APC. Science 275: 1787-1790, 1997.

27. Wang W, Xiao X, Chen X, Huo Y, Xi WJ, Lin ZF, Zhang D, Li YF, Yang F, Wen WH, et al: Tumor-suppressive miR-145 co-repressed by TCF4- $\beta$-catenin and PRC2 complexes forms double-negative regulation loops with its negative regulators in colorectal cancer. Int J Cancer 142: 308-321, 2018.

28. Ishiguro H, Wakasugi T, Terashita Y, Sakamoto N, Tanaka T, Sagawa H, Okubo T and Takeyama H: Nuclear expression of TCF4/TCF7L2 is correlated with poor prognosis in patients with esophageal squamous cell carcinoma. Cell Mol Biol Lett 21: 5, 2016.

29. Zhao W, Zhou J, Deng Z, Gao Y and Cheng Y: SPOP promotes tumor progression via activation of $\beta$-catenin/TCF4 complex in clear cell renal cell carcinoma. Int J Oncol 49: 1001-1008, 2016.

30. Zhang Q, Gao M, Luo G, Han X, Bao W, Cheng Y, Tian W, Yan M, Yang G and An J: Enhancement of radiation sensitivity in lung cancer cells by a novel small molecule inhibitor that targets the $\beta$-catenin/Tcf4 interaction. PLoS One 11: e0152407, 2016.

This work is licensed under a Creative Commons Attribution-NonCommercial-NoDerivatives 4.0 International (CC BY-NC-ND 4.0) License. 